\title{
Serratus plane block impact in pain and opioid uptake in oncological breast surgery.
}

\author{
Casasempere Sanus A., Mazzinari G., Ortega Monzó J.M., Rovira Soriano L., Belaouchi M., Esparza Miñana J.M. \\ Hospital de Manises, Dept of Anaesthesiology, Manises, Spain
}

\section{INTRODUCTION}

Regional anaesthesia is related with less incidence of cancer recurrence and less chronic pain development ${ }^{1-2}$. The paravertebral block is the most common regional technique in breast surgery, but it is difficult to perform and is not free from complications ${ }^{3}$.

Alternatively, ultrasound guided serratus plane block achieves complete analgesia of the axillomammary area by reaching the nerves between the pectoral and the serratus muscles (pectoral nerves, supraclavicular and long thoracic nerve) with a high security profile ${ }^{4-5}$. The goal of this study is to evaluate the effect of the serratus plane block on opioids requirements during surgery and the immediate postoperative period as well as on the level of postoperative pain in oncological breast surgery.

\section{MATERIALS AND METHODS}

A randomized intervention study is presented (NCT02905149). Patients recruited undergoing oncological breast surgery are assigned to Control (intravenous opioid) or Serratus group. Every patient receives general anaesthesia with opioid analgesia according to the current hospital protocol. The block is performed with $20 \mathrm{ml} 0^{\prime} 5 \%$ Levobupivacaine immediately after induction. In the postoperative period every patient is given a morphine PCA infusion pump which allows boluses of $1 \mathrm{mg}$ every $15 \mathrm{~min}$ but no continuous infusion. Patients are followed up during $24 \mathrm{~h}$, asked for opioid consumption and pain estimated by VAS (visual analogue scale). We analyzed differences in opioid uptake in $24 \mathrm{~h}$ and pain VAS scores at set intervals $(1,3,6,12,24 \mathrm{~h})$.
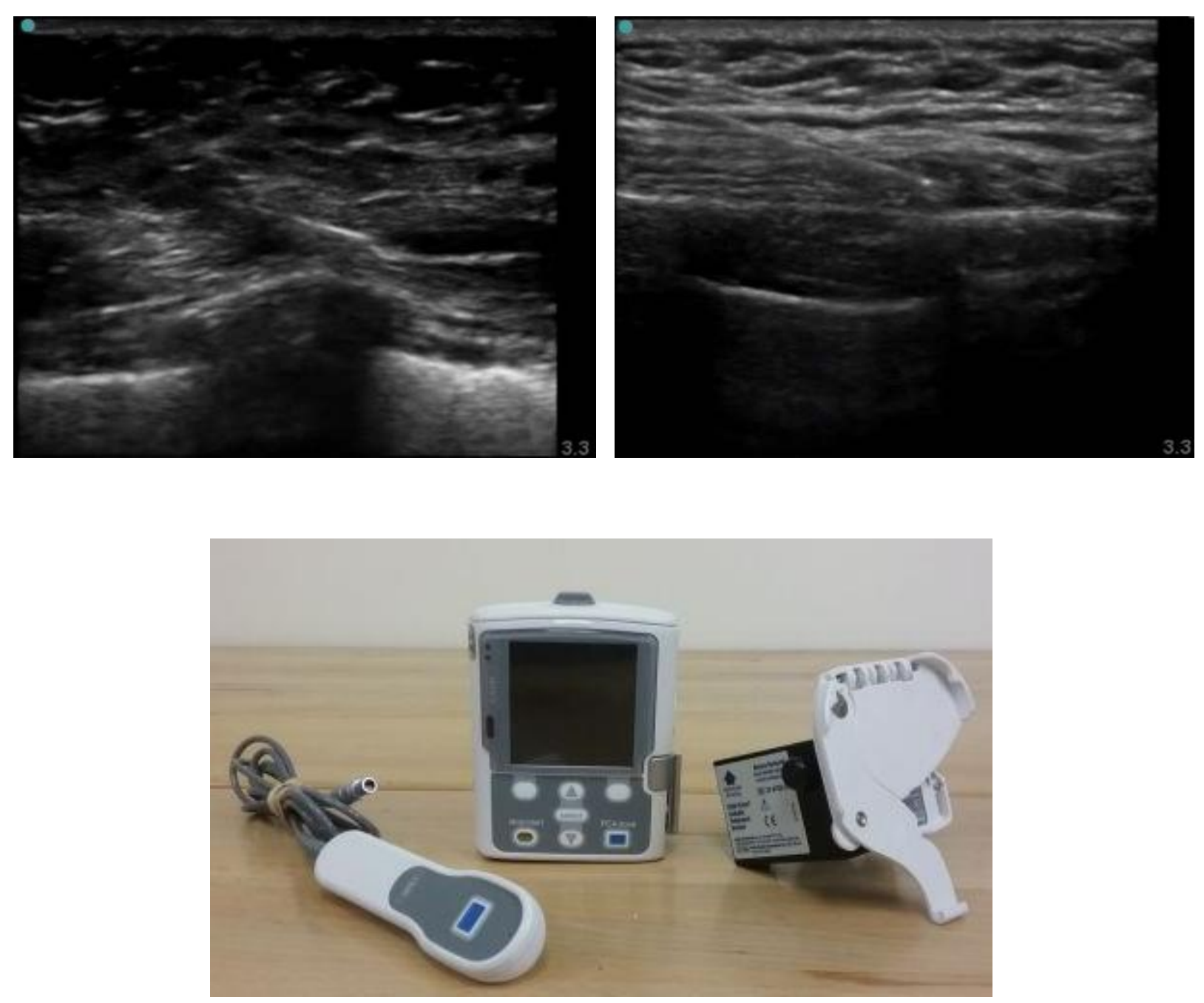

\section{RESULTS AND DISCUSSION}

We offer preliminary results from the data analysis of the first 11 recruited patients. The serratus plane block was effective in all cases. The cumulative opioid consumption over $24 \mathrm{~h}$ was higher in control group $(35.2 \mathrm{mg}$, SD 5.96, 95\% CI 21.92,48.48) than in Serratus group $(21.5 \mathrm{mg}$, SD 3.41, 95\% CI 13.89, 29.11) (Fig 1). VAS scores for $24 \mathrm{~h}$ cumulative pain at rest and movement were higher in control than in Serratus group (AUCRcontrol 54.3, AUCRserratus 7.08, AUCMcontrol 75.9, AUCMserratus 13.3) (Fig 2). Serratus group pain VAS scores were 2 or more points lower than the control group scores, both at rest and at movement (Fig 3).

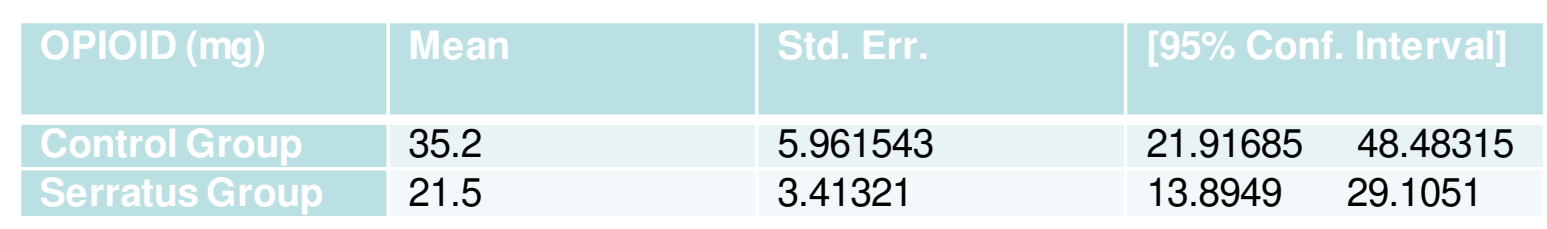

Fig 1. Cumulative opioid uptake during $24 \mathrm{~h}$ postop.

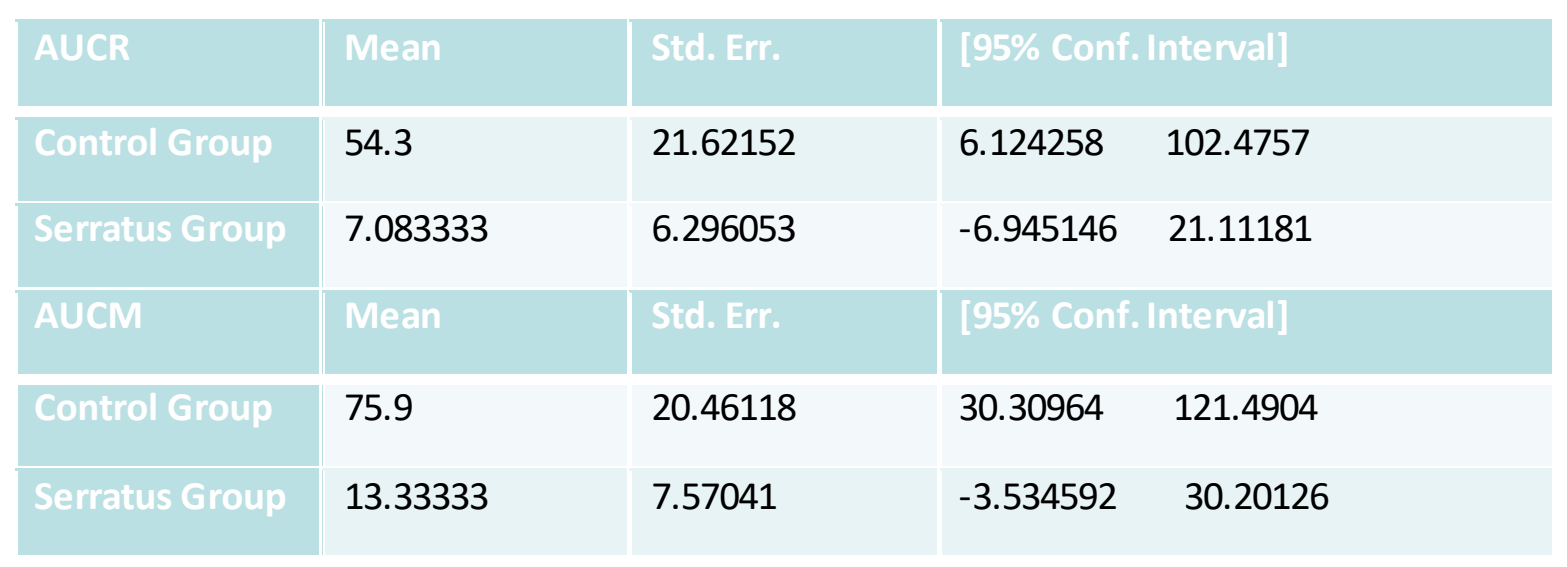

Fig 2. VAS scores. Cumulative pain during $24 \mathrm{~h}$ postop.

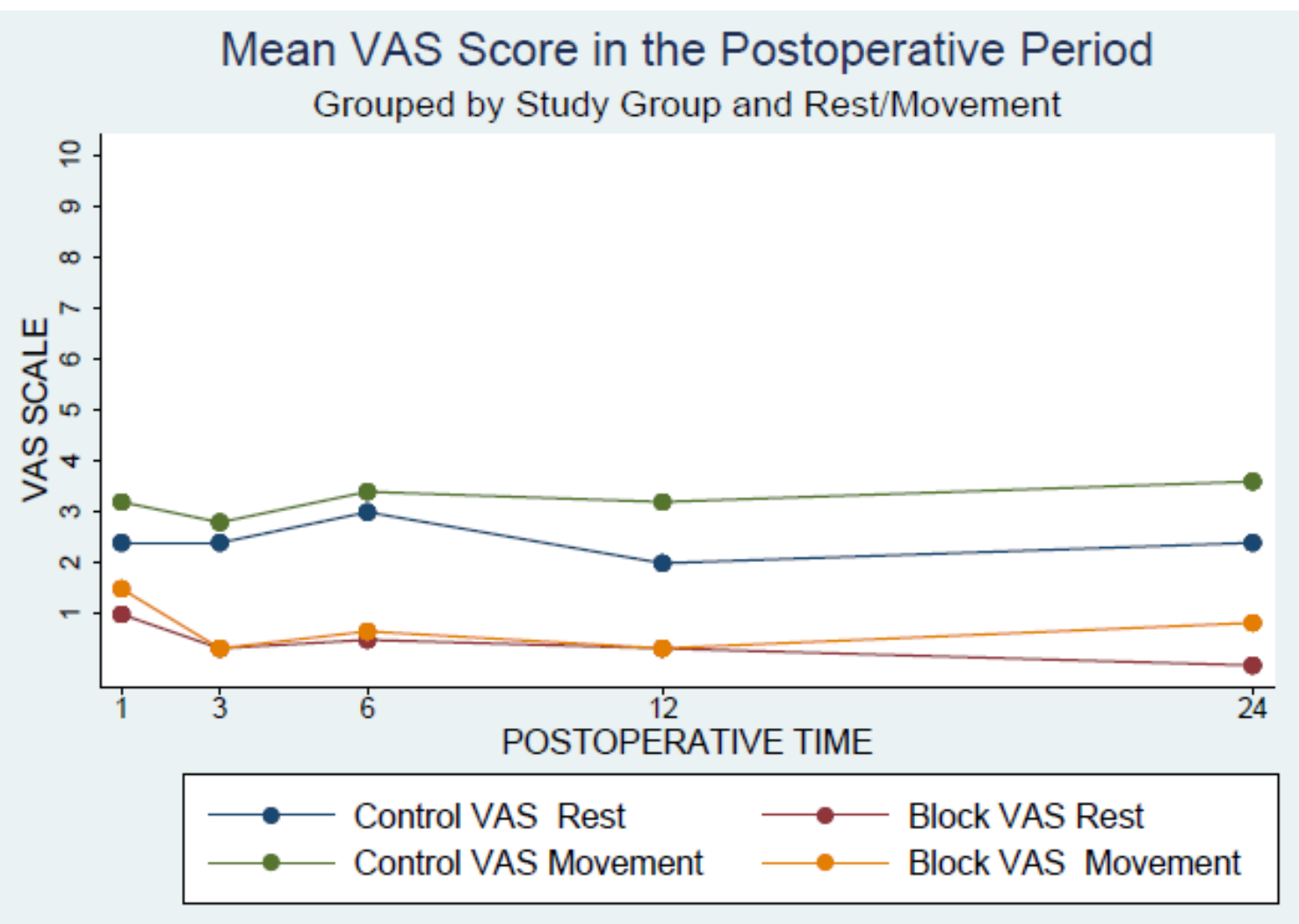

Fig 3.VAS scores.

\section{CONCLUSION}

Serratus plane block provides adequate intraoperative and postoperative analgesia for oncological breast surgery. It is a simple, reproducible and safe technique, and may be an alternative to opioid analgesia or paravertebral block.

Andreae MH, Andreae DA. Regional anesthesia to prevent chronic pain after surgery after surgery: a Cochrane systematic review and meta-analysis. Br J Aneaesth 2013;111(5):711-20.

Ash SA, Buggy DJ. Does regional anaesthesia and analgesia or opioid analgesia influence recurrence after primary cancer surgery? An update of available evidence. Best Pract Res Clin Anaesthesiol. 2013 Dec;27(4):441-56 Schnabel A, Reichl SU, Kranke P, Pogatzki-Zahn M, Zahn PK. Efficacy and safety of paravertebral blocks in breast surgery: a meta-analysis of randomized controlled tirals. Br J Anaesthesiol 2010;105(6): 842-52. Blanco R, Parras, McDonnel JG, Prats.Galino A. Serratus plane block: A novel ultrasound guided thoracic wall nerve block. Anaesthesia 2013;68:1107-1113.

Fajardo Pérez M, García Miguel FJ, López Álvarez S, Dieguez García P, Alfaro de la Torre P. Analgesic combined lateral and anterior cutaneous branches of the intercostal nerves ultrasound block in ambulatory breast surgery. Cir May Amb 2012;17(3): 95-1 